\title{
THE CIRCULAR ECONOMY AS AN ENGINE FOR REGIONAL AND URBAN DEVELOPMENT - THE NEW PARADIGM OF THE 21ST CENTURY
}

DOI: http://dx.doi.org/10.18509/GBP.2020.75

UDC: 332.14:338.121(4-672EU)

711.4:338.121(4-672EU)

\section{Metodi Ivanov}

Sofia University „St. Kliment Ohridski“, Faculty of Geology and Geography, Department "Regional Development", Sofia, Bulgaria

\begin{abstract}
The perception of the circular economy as an engine for regional and urban development on the part of the European Union is the result of the perception that all cities and municipalities will be directly involved in the transition to a circular economy, since on the one hand, local authorities have a clear idea of the challenges and opportunities at local level, necessitating the development of own development strategies based on the possibility of implementing integrated territorial investments, combined with funding and planning local initiatives to shift towards a circular economy. The transition to a circular economy necessitates a change in the thinking and perception of existing systems in individual cities and regions, with the development, through the cooperation of a circular model of the economy of the individual regions in the European Union, in which the model would include all stakeholders, as well as the business community - in the face of small and medium-sized enterprises in less developed regions and public sector organizations to support the development of local markets by supporting projects innovations and modern information and communication technologies. The presentation of the circular economy as an engine for regional and urban development in the $21 \mathrm{st}$ century is based on the perception that this process relies on both cohesion policy and smart specialization and synergies with European structural and investment funds to stimulate circular economy markets. Attention must be paid both to the macro-regional approach to regional co-operation and coordination in the creation of regional markets and to cross-border and trans-national co-operation programs to support interregional cooperation in the area of circular economy activities in order to transform cities and regions on the territory of Bulgaria, as well as other lagging regions on the territory of the European Union in competitive on the world markets.
\end{abstract}

Keywords: circular economy, regional economic, urban development, regional development

\section{INTRODUCTION}

The presentation of the circular economy as an engine for regional and urban development is due to the continuing rapid growth of resource consumption and the increasing environmental impact, which has made it necessary to take the circular economy as the new paradigm of the 21 st century. In the current century, the world is facing great uncertainty about the pace of development of the global economy, due to the increasingly scarce resources, resulting in the view of individual countries to seek opportunities to improve resource efficiency throughout the economy. Businesses are also reorienting themselves to seeking ways to reuse manufactured products or their components and 
recover inputs in production, which can be achieved by improving recycling opportunities and effectively utilizing the residual value gained from reusing resources in the system production and exchange, in which, at each stage of the product life cycle, manufacturers strive to increase resource efficiency they and simultaneously reducing harmful effects on the environment. The notion that the circular economy provides the basic guidelines to significantly reduce and sustainably depend on the economy's resource dependency and to move towards the scarcity of non-renewable natural resources is the result of the ability of the circular economy to offer solutions, especially for enhancing production skills and design, as well as developing and building new business models based on crossindustry collaboration. Perception as a paradigm can be the result of the perception that the circular economy is perceived as a closed cycle that covers each of the three spheres, namely offering and building responsible choice for producers, demand and consumer behavior and naturally building a responsible management system of waste. In order to increase resource efficiency, the European Commission takes the notion of a circular economy as a priority. „By 2050 the EU's economy has grown in a way that respects resource constraints and planetary boundaries, thus contributing to global economic transformation. Our economy is competitive, inclusive and provides a high standard of living with much lower environmental impacts. All resources are sustainably managed, from raw materials to energy, water, air, land and soil. Climate change milestones have been reached, while biodiversity and the ecosystem services it underpins have been protected, valued and substantially restored.[1]" Here it outlines the key task set by the European Commission, which is to turn the European economy into a resource efficient, environmentally friendly and competitive low carbon economy by creating job opportunities, securing growth by increasing investment, combating climate change changes, as well as the introduction of industrial innovation into modern industries. Particularly given that a circular economy means the concept of a system of production and consumption that generates the least possible loss, whereby everything is reused, recycled or used for the production of other output goods and services. By seeking and creating the opportunity to process products and modernize production processes, it will help to minimize the losses in search of economic, environmental and social impact of the use of the circular economy in the current 21st century. By pursuing the objective of structuring a circular economy to function as a kind of closed system in the production of goods and services by limiting the use of raw materials and energy on the one hand and reducing the generation of waste on the other. The main objective of the circular economy is to create an opportunity to increase resource efficiency while reducing harmful environmental impacts in the search for opportunities to increase consumer well-being. In the 21 st century, the main theme in the production of companies has been the creation of competitive and sustainable production, which at the same time be environmentally friendly by increasing resource efficiency, which will simultaneously reduce production costs and increase productivity. It is the modern companies that are focusing their efforts on waste recovery, on the re-use of waste, as well as on their actual containment. The progress made in the application of eco-innovation provides businesses with the opportunity to create new products, use new processes in production through the introduction of new technologies and the application of organizational structures that enable the transition to 21st century recycling-based business models, repair, reuse of products or their components. The proliferation of the "circular economy" concept, which seeks maximum utilization of resources, is an opportunity to achieve a new type of economic growth that will create additional jobs, while at the same time creating the 
opportunity to address the socially significant challenges posed by the exhaustion of natural resources and their ever-increasing prices on international, regional and even national markets. The cyclical nature of economic development implies the emergence of a new economic and financial crisis, which will further limit the financial sector's ability to provide funding for the real economy and make long-term investments, which will be combined with a decline in investor confidence and will again reduce financing for all sectors of the economy will be particularly strong in developing countries as well as in the less economically developed countries in the European union.

\section{THE CIRCULAR ECONOMY IN THE FUTURE DEVELOPMENT OF THE EUROPEAN UNION}

"The EU's target of reducing greenhouse gas emissions by $80-95 \%$ by 2050 will require fundamental changes not only in energy, food and mobility systems, but also in the way raw materials and manufactured products are produced, traded, used, maintained and fed back into the economy at the end of their life.[2]". In the future development of the European Union, the realization of the circular economy concept will be based on the creation of financing mechanisms that are adapted to existing business models, in order to bring out the specific barriers to the creation of economic growth based on the application of the circular economy. It is precisely the role of the European Union institutions and the governments of the individual countries in applying the concept of a circular economy that will be based on the creation of opportunities to overcome barriers to entry and to create market niches based on the understanding of the application of business models based on the concept of a circular economy where new practices can be developed and improved. In this way, the conditions for realizing the effective potential of product service systems must be taken into account. In the next figure will see the system barriers, reinforcing trends, conditions for improved product circularity (Figure 1).

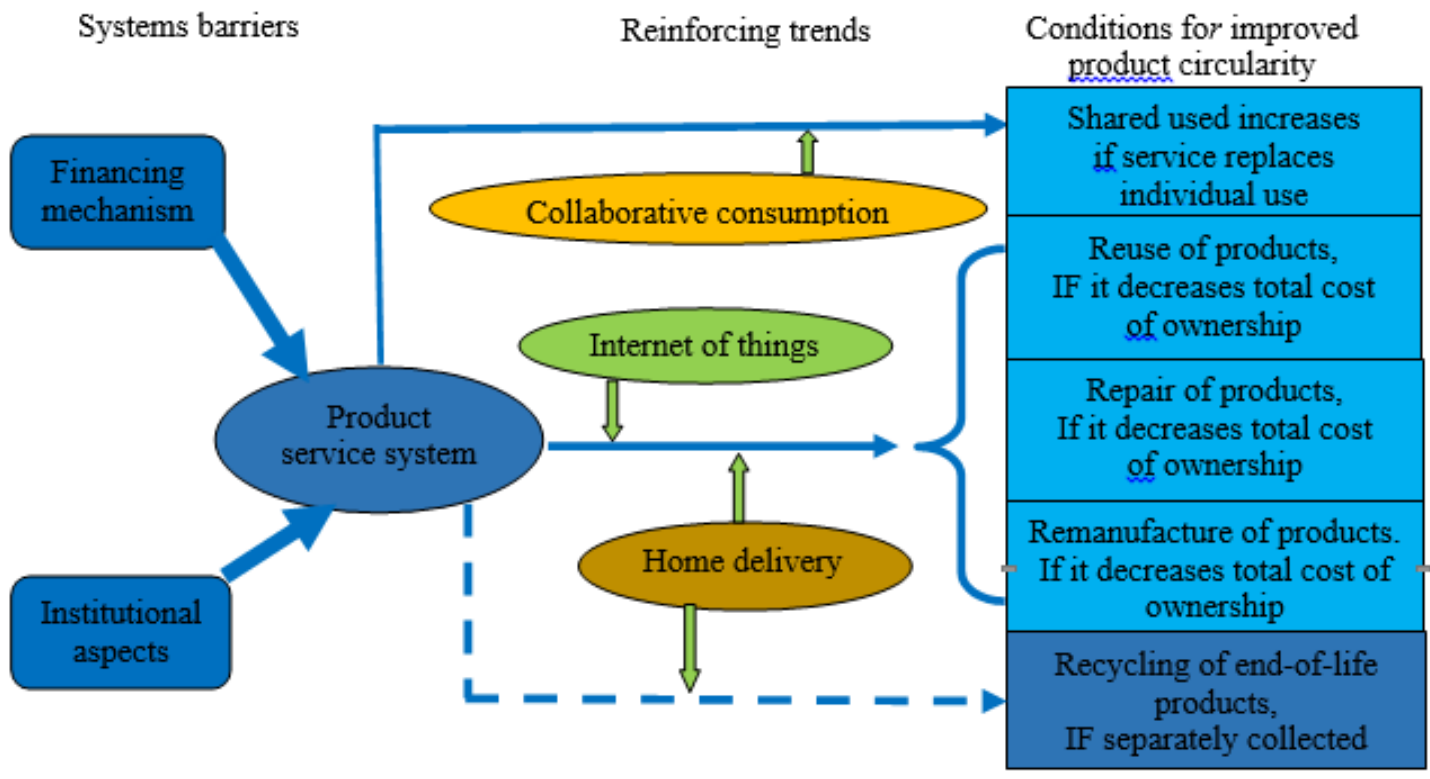

Figure 1. Enabling product circularity with product-service systems Source: Circular by design, Products in the circular economy, ISSN 1977-8449

In order to carry out the process of introducing the circular economy model in individual countries, the application of a complex approach should be sought, since different entities 
are involved in the system, and especially given the interdisciplinary nature of this concept, it should be expected that its implementation will be linked to a number of difficulties, such as the reporting of results and the lack of synthetic indicators at regional and local level, although implemented by the European Commission and reported by Eurostat and circular economy. But a major problem for developing countries such as Bulgaria is the lack of macro and micro level information and data, as well as the lack of widespread publicity of good practices and opportunities that new business models offer to achieve balanced regional sustainable development based on the application of the concept of a circular economy. In addition, an obstacle to the expansion and implementation of the circular economy concept is the country's established vertical structure of our industrial system, which is also characteristic of other developing countries, where inter-sectoral links are also lacking or poorly developed. Since in order to strengthen the implementation of the concept of a circular economy, it is important to strengthen the interaction between different industries and sectors of the economy, thereby realizing the re-use of components and recycled materials as secondary raw materials in the life cycle stages of the manufactured products. The introduction of a systematic approach to the implementation of the concept of a circular economy and the implementation of complex cross-sectoral links, as well as the availability of management skills are the basic elements for achieving success based on the circular economy. On the other hand, barriers also create financial constraints, given the high share of small and medium-sized enterprises in the country, the implementation of the circular economy concept must be linked to serious investments in new resource-saving technologies, for which a large part of small and medium-sized enterprises medium-sized enterprises are not available, which necessitates the search for new financial mechanisms, both at national and European Union level, especially for countries such as Bulgaria and developing countries. „These circular business models lead to the creation of a circular economy concept. The concept of circular economy sets a set of principles for production and consumption, radically different from the take-make-dispose linear regime prevailing in today's market economies, based on continued economic growth and increased resource productivity.[3]"“

\section{CHALLENGES TO THE IMPLEMENTATION OF THE CIRCULAR ECONOMY CONCEPT IN BULGARIA.}

The identification of the challenges to the implementation of the concept of a circular economy in the country must be linked to the definition of the role and involvement of all stakeholders, and from the state perspective, it must develop policies while ensuring the framework conditions, the economic operators can have confidence in the realization of their investment intentions. While companies are tasked with reorganizing their supply and distribution schemes to achieve circularity and resource efficiency, and in order to achieve the concept, the circular economy model needs to become a national priority, since conceptually pursues the implementation of the model, not only in selected significant sectors, such as increasing energy efficiency and reducing emissions, but also seeking the opportunity to reduce waste and increase to share their recycling, which is accompanied by extending the product life cycle. Consumers also have a role in the implementation of the concept, which has to change their attitude towards the products and their attitudes towards the relationship "consumer", "user", "sharing" the product or the service bringing satisfaction. There is definitely a need for an information campaign to address traditional consumer habits as they can become a barrier to the development 
of new products and services resulting from the application of the circular economy. On the other hand, the state should stimulate and encourage investments in innovations for the circular economy and their implementation by companies, since by stimulating private financing and taking part of the investment risk through the implementation of publicprivate partnerships regarding the scarcity of resources and the use of recycled products and components in new industries. On the other hand, the state also has another mechanism for stimulating the investment activity of companies, such as the implementation of an eco-fiscal policy, which can be used to give signals for investing in resource efficiency by eliminating environmentally harmful subsidies and transfer of taxation from labor to pollution and resources. Investment in innovation helps reduce environmental pollution, supports the deployment of products whose production consumes less resources, while improving resource efficiency, which can lead to economic growth that will lead to and to increase employment and to overcome the dependence on economic growth that results from inefficient use of resources and increased pollution. The implementation of the circular economy model can reduce energy consumption by creating sustainable and connected smart cities of the future, in which a large amount of waste is reused. Given that the focus of the circular economy concept is on environmental attitudes and environmental compliance, it is definitely up to each country to focus its efforts on promoting low hydrocarbon production as well as reducing gas production with greenhouse effect. Individual countries should prioritize funding for research projects in the field, the creation of more and more intelligent electrical systems, and stimulate private investment, by co-financing initiatives related to the reuse, sharing and extension of the product life cycle. In this context, individual countries should set specific targets in the medium term, such as reducing the share of waste that is landfilled or increasing the share of recycled plastic waste, or the gradual replacement of certain plastic products with organic substitutes. Achieving such goals can be aided by the use of financial instruments available to the state, namely the determination of the amount of municipal waste tax and the landfill tax. Or by introducing regulations that require manufacturers to demonstrate and demonstrate the extended life cycle of the product they offer, as well as the replacement parts available. Definitely businesses shift to a circular model brings economic benefits but companies should be encouraged, as well as in co-financing initiatives related to technological upgrading or buying a new resource-saving technologies and the introduction of non-waste technology. On the other hand, the executive should be committed to the widespread promotion and launch of campaigns, initiatives and actions related to the implementation of good practices in the area of environmental responsibility, as well as providing incentives for business. Of course, adequate institutional monitoring and control mechanisms must also be put in place to ensure the implementation of environmental standards, as well as to encourage the transition to practices based on the principles of the circular economy. The state must also commit itself to developing a focused investment policy that is geared to the needs of preventing and overcoming the environmental, natural and natural disasters arising from the production activity of companies.,,Floods, droughts, earthquakes, and others have accompanied human development from its beginning. That is why disaster risk reduction and enhancement of social sustainability remain key challenges for both developing and developed countries. The main reason for that is the growing exposure of humans and their assets in risk areas on the one hand, and on the other, the increased frequency of extreme natural events due to climate change[4]". 


\section{CONCLUSION}

The implementation of the circular economy model is slowly, with transformation extremely difficult, and some of the main obstacles are the lack of awareness of managers and entrepreneurs, which is complemented by the conservative attitude of some of the business, as a result of fear of the new and the unknown. On the other hand, business often bets securely, which is why there is no desire for radical change, especially in terms of devoting resources to technological renewal and the application of new green and resource-saving technologies. On the other hand, the lack of trained and motivated staff is a problem for the business. Often, there is a lack of basic information and capacity to move to the circular economy solutions, as the benefits can be linked to the creation of products with improved performance and extended life. The transition to a circular economy, on the other hand, requires the development of a viable strategy that incorporates long-term goals, while at the same time presenting and promoting the idea of an eco-concept related to the creation of longer-life products, while being reusable, repair or recycling. Promoting good practices should play an important role in the development of the strategy, as they will have a stimulating effect on the business to make a change in the production models used. On the other hand, sectoral and professional organizations are not sufficiently used, since adopting specific quantitative targets for the use of a specific volume of recycled materials can encourage producers to increase their responsibility in the implementation of environmentally friendly industries. Another approach is to revise municipal waste management policy, which should be accompanied by the development of appropriate schemes at regional level that will have a multiplier effect in the transition to a circular economy. The need to successfully integrate eco-fiscal instruments to ensure fiscal neutrality will contribute to increasing the level of waste reuse in the production process, providing additional financial incentives for environmentally sustainable projects that to conserve nature and reduce the usability of natural resources, while at the same time creating the opportunity to extend the life cycle of products that the process involves and creates repair and recycling conditions. Certainly the implementation of the circular economy concept implies an increase in the amount of investments in key resources and natural capital in order to achieve future economic growth through the circular economy concept, both locally and internationally. The result of the implementation of the circular economy concept can be when a combination of market and regulatory instruments is implemented, which involves the introduction of environmental taxes, consideration of the possibility of eliminating environmentally harmful subsidies, and last but not least an opportunity to mobilize free public and private financial resources towards investments to increase people's skills and create new green jobs. The implementation of the circular economy model must be geared towards achieving radical change, both in production and consumer behaviors, in accordance with territorial planning and regional development. The road to achieving a sustainable circular economy requires combining ambitious environmental goals by maintaining stable social requirements and accountability to future generations based on the development and implementation of a consistent legal framework aimed at achieving and maintaining sustainable production and consumption. ,The development and growth of the autonomy of the regions implies an increase in their degree of recognition, related to the increase of international economic relations and trade. One of these options is free economic zones, as a particular type of state (or private) regulation of international economic relations and trade. Free economic zones around the world must include free - commercial, port, economic, banking - or duty - free shops and warehouses. 
Generally, they are designed to stimulate industrial exports, increase foreign exchange earnings, and workplaces used as "laboratories" to test new economic methods and mechanisms. Another favorable feature of all free economic zones is the favorable investment climate - customs, tax, financial privileges and administrative concessions compared to the general regime operating in the rest of the economic area in one or another country (respectively region). Often, factors such as political stability, investment guarantees, infrastructure quality, and workforce qualifications are crucial. The localization of such a free zone is an essential element of the national strategy and an important approach in the development of regional policy for a given territory. This is one of the world-wide recognized forms of organizing production, especially of high-tech products, as well as creating conditions for providing modern technologies, as well as for moving foreign direct investments and accelerating the development of certain economic regions. Free economic zones are an important tool for regional development.[5]“"

\section{REFERENCES}

[1] EUROPEAN COMMISSION, Roadmap to a Resource Efficient Europe , Brussels, 20.9.2011 $\operatorname{COM}(2011) \quad 571$ final available to https://www.europarl.europa.eu/meetdocs/2009_2014/documents/com/com_com(2011)0571_/c om_com(2011)0571_en.pdf on 01.02.2020.

[2] Circular by design, Products in the circular economy, ISSN 1977-8449, available to https://www.eea.europa.eu/publications/circular-by-design on 01.02.2020

[3] Naydenov, K1., Circular tourism as a key for eco-innovations in circular economy based on sustainable development, 18th International Multidisciplinary Scientific GeoConference SGEM2018, DOI: 10.5593/sgem2018/5.3/S28.017

[4] Zlatunova, D., Babukova, P., Integrated vulnerability analysis for disasters risk reduction, International Scientific Conference GEOBALCANICA, 2016, DOI: 10.18509/GBP.2016.22, available https://www.researchgate.net/publication/307940747 INTEGRATED VULNERABILITY AN ALYSIS FOR DISASTERS RISK REDUCTION on 02.02.2020.

[5] Naydenov, Kl., The role of the free economic zones for the development of the regional economy, 5th SGEM International Multidisciplinary Scientific Conferences on SOCIAL SCIENCES and ARTS SGEM2018, DOI: 10.5593/sgemsocial2018/5.2/S20.069 